\title{
VOLUNTARY PROVISION OF PUBLIC GOODS FOR BADS: A THEORY OF ENVIRONMENTAL OFFSETS
}

\author{
Matthew J. Kotchen \\ Working Paper 13643 \\ http://www.nber.org/papers/w13643

\section{NATIONAL BUREAU OF ECONOMIC RESEARCH} \\ 1050 Massachusetts Avenue \\ Cambridge, MA 02138
}

November 2007

I am grateful for helpful comments I received from an anonymous reviewer and seminar participants at Brown, Harvard, Stanford, Vanderbilt, and the NBER. The views expressed herein are those of the author(s) and do not necessarily reflect the views of the National Bureau of Economic Research.

(C) 2007 by Matthew J. Kotchen. All rights reserved. Short sections of text, not to exceed two paragraphs, may be quoted without explicit permission provided that full credit, including $\odot$ notice, is given to the source. 
Voluntary Provision of Public Goods for Bads: A Theory of Environmental Offsets

Matthew J. Kotchen

NBER Working Paper No. 13643

November 2007

JEL No. H0,H41

\begin{abstract}
$\underline{\text { ABSTRACT }}$
This paper examines voluntary provision of a public good that is motivated, in part, to compensate for other activities that diminish the public good. Markets for environmental offsets, such as those that promote carbon neutrality to minimize the impact of climate change, provide an increasingly salient example. An important result, related to one shown previously, is that mean donations to the public good do not converge to zero as the economy grows large. Other results are new and comparable to those from the standard model of a privately provided public good. The Nash equilibrium is solved explicitly to show how individual direct donations and net contributions depend on wealth and heterogenous preferences. Comparative static analysis demonstrates how the level of the public good and social welfare depend on the technology, individual wealth, and an initial level of the public good. Application of the model in an environmental context establishes a starting point for understanding and making predictions about markets such as those for carbon offsets.
\end{abstract}

Matthew J. Kotchen

Bren School of Environmental Science \& Management

and Department of Economics

University of California, Santa Barbara

Santa Barbara, CA 93106-5131

and NBER

kotchen@bren.ucsb.edu 


\section{Introduction}

Why do individuals voluntarily provide public goods? The question has received much attention in the economics literature, and two general answers have emerged. The first is based on a standard model of private provision of a pure public good. Agents are assumed to benefit from the aggregate level of a public good and thus have some incentive for private provision. While the additional incentive to free ride ensures that the equilibrium level of the public good will fall short of the Pareto-efficient level, the theory nevertheless predicts some degree of voluntary provision. ${ }^{1}$

The second explanation has grown out of the need to reconcile why observed levels of private provision regularly exceed those that the standard theory predicts. Research in this area can be characterized broadly as refinements or extensions on the impure public good model. The general approach is to assume that agents obtain a private benefit from some aspect of their own provision, and this encourages provision beyond that which would occur if benefits came from only the public good itself. Different interpretations of the private benefit range from a feeling of "warm-glow" satisfaction, social approval, prestige, and signaling about income. ${ }^{2}$

The present paper pursues a further explanation for voluntary provision of a public good. The idea is that provision may occur, in part, to offset other activities that have an adverse effect on a public good. Consider an activity that produces a negative externality through diminishment of a public good. Assuming the individual cares enough about the public good, she may seek to minimize her adverse effect on it. One possibility is for the individual to voluntarily restrain her behavior. ${ }^{3}$ Another possibility, which need not be mutually exclusive, is for the individual to offset some (or all) of her detrimental effect through direct provision of the public good.

Examples of voluntary provision motivated in this way are increasingly common in an environmental context. Perhaps the most prominent example comes from the increased popularity of carbon offsets. Individuals and institutions that are concerned with their emissions of carbon dioxide, which contribute to climate change, have opportunities to make direct donations that help reduce emissions

\footnotetext{
${ }^{1}$ Samuelson $(1954,1955)$ is widely recognized for his pioneering work on this model. More recent and standard references that employ a game-theoretic approach include Bergstrom, Blume and Varian (1986), Andreoni (1988), and Cornes and Sandler (1996).

${ }^{2}$ Cornes and Sandler $(1984,1994,1996)$ develop the general impure public good model, and references that are described here as refinements or extensions include Glazer and Konrad (1986), Hollander (1990), Andreoni (1990), Harbaugh (1998), and Kotchen (2005).

${ }^{3}$ See Kotchen and Moore (in press) for empirical evidence of voluntary restraint with respect to household electricity consumption and air pollution emissions.
} 
elsewhere. Currently, there are at least 53 nonprofit and commercial organizations that specialize in providing opportunities for carbon offsets, which typically involve investments in renewable energy and reforestation programs. ${ }^{4}$ While interest in purchasing carbon offsets is clearly on the rise, participants in the market already include individuals, governments, and industry. ${ }^{5}$

It is easy to conceive of other, more general, charitable activities as being motivated with the idea of an offset in mind. Anecdotal examples include a real estate developer who donates land for a park, or a shrewd investor who makes a large donation to clear his conscience. It is also the case that acts in violation of one's religion that require monetary penance, such as the forbiddance of gambling by Islam, are consistent with the notion of donations as an offset.

This paper is not the first to consider private provision of a public good to offset a bad. Vicary (2000) modifies the impure public good model to account for an impure public bad, that is, an activity that diminishes the level of a public good. His model also permits individuals to make direct donations to the public good as a compensating mechanism. The model is then used to answer the question of what happens to donations in a large economy. Vicary finds, contrary to the standard model with a pure public good, that a substantial proportion of the population can be donors in an economy with many individuals. This result is useful because it provides a theoretical basis for donations that more closely matches the empirical evidence.

The model developed here begins with the same basic idea but, in contrast, generalizes the pure public good model. This more parsimonious setup has the advantage of making comparisons with the standard model more direct and intuitive. While the results for donations in a large economy are closely related to those in Vicary (2000), other results are entirely new and relate to a different set of questions: How will direct donations and net contributions depend on wealth and heterogenous preferences? How will changes in the exogenous variables - technology, wealth, and the ambient level of the public good - affect the equilibrium level of the public good? And how will changes in these same exogenous variables affect social welfare?

On one level the paper makes a general contribution to the literature on pri-

\footnotetext{
${ }^{4} \mathrm{~A}$ directory of carbon offset providers can be found online at the following url: www.carboncatalog.org/providers.

${ }^{5}$ The New York Times describes the concept behind Terrapass, one of the for-profit companies that offers carbon offsets, as one of the most influential ideas of 2005 (Dominus, 2005), and the company now sells offsets for automobile and air travel. The British government purchased carbon offsets for air travel required by all participants at the G8 Summit in 2005. Many companies and organizations are using offsets to reduce emissions and pursue a carbon neutral policy; examples include The Rockefeller Brothers Fund, Ben \& Jerry's, Green Mountain Coffee Roasters, Timberland, the Rolling Stones, and the Dave Matthews Band.
} 
vate provision of public goods. The setup of the model is novel, and many of the results are derived using a new approach, developed by Cornes and Hartley (2007), for analyzing aggregative public good games. On another level the paper makes a contribution through its application to environmental offsets, which is used throughout as a motivating and explanatory example. Specific predications are made about the extensive and intensive margins of voluntary environmental offsets, such as those for carbon dioxide emissions. The results are also informative about the ways in which changes in the technology of a polluting good will affect the purchase of offsets, environmental quality, and social welfare. Some of the results are rather counterintuitive and are shown to have a similar structure to that in Cornes and Sandler (1989) and Kotchen (2006). For example, it is shown how a technology improvement that makes a polluting good more environmentally friendly can actually diminish both environmental quality and social welfare. Finally, it is shown that in larger economies, environmental offsets will always persist, as compensating for consumption of the polluting good becomes even more important.

\section{The Model}

Assume there are $i=1, . ., n$ individuals in the economy. Each individual has preferences according to a strictly increasing and strictly quasiconcave utility function $U_{i}=U_{i}\left(x_{i}, G\right)$, where $x_{i}$ is a private good and $G$ is a public good, which can be interpreted as environmental quality. Individuals are able to consume the private good and directly provide the public good; however, a key feature of the model is that consumption of $x_{i}$ is generally not independent of $G$. In particular, consumption of each unit of $x_{i}$ causes a reduction in $G$ of $\beta \geq 0$ units. Letting $y_{i}$ denote each individual's "direct provision" of $G$, it follows that each individual's "net contribution" can be written as $g_{i}=y_{i}-\beta x_{i}$. Aggregate provision of the public good is assumed to be $G=\sum_{i=1}^{n} g_{i}+\bar{G}$, where $\bar{G}>0$ is an initial level of the public good, i.e., ambient environmental quality.

Each individual is endowed with exogenous wealth $w_{i}$, and normalizing all prices to unity, individual $i$ 's utility maximization problem can be written as

$$
\begin{gathered}
\max _{x_{i}, y_{i}} U_{i}\left(x_{i}, G\right) \\
\text { subject to } x_{i}+y_{i}=w_{i}, G=\bar{G}+G_{-i}+y_{i}-\beta x_{i},
\end{gathered}
$$

where $G_{-i}=\sum_{j \neq i} g_{j} .{ }^{6}$ It is convenient to rewrite the utility maximization problem with an implicit choice over the aggregate level of $G$. Use the second con-

\footnotetext{
${ }^{6}$ Note that if $\beta=0$ and we rescale so that $\bar{G}=0$, the setup of the model is equivalent to the standard model of private provision of a pure public good.
} 


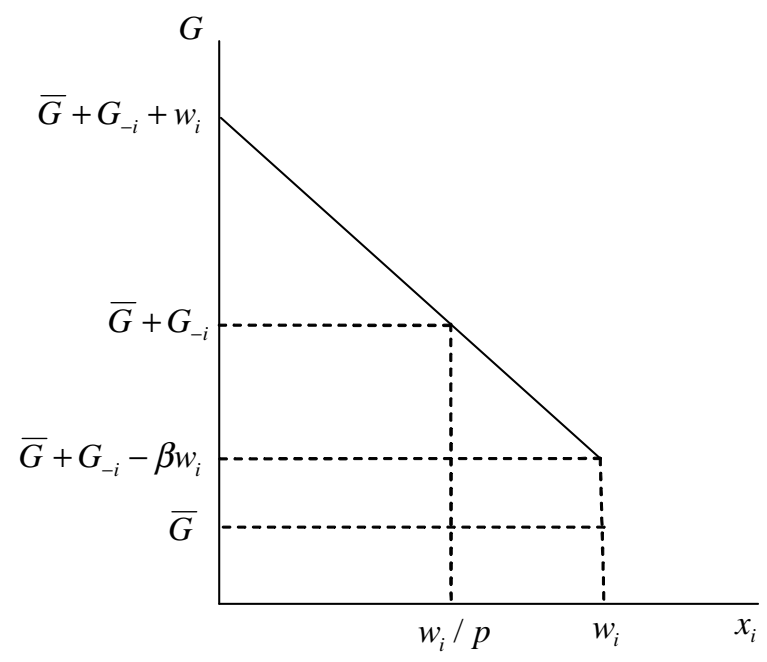

Figure 1: An individual's budget set

straint in (1) to substitute $y_{i}$ out of the first constraint, and rearrange to get $p x_{i}+G=w_{i}+\bar{G}+G_{-i}$, where $p=1+\beta$ is the price of $x_{i}$ in terms of $G$. This constraint is referred to as the individual's "full-income" budget constraint because it accounts for personal wealth plus the value of exogenously given levels of the public good. Using the new constraint, (1) can be rewritten as

$$
\begin{gathered}
\max _{x_{i}, G} U_{i}\left(x_{i}, G\right) \\
\text { subject to } p x_{i}+G=w_{i}+\bar{G}+G_{-i}, G \geq \bar{G}+G_{-i}-\beta w_{i} .
\end{gathered}
$$

Figure 1 illustrates one possibility for the individual's budget set. The frontier is linear with slope $-p$. The feasible set includes quantities of $G$ that are below the exogenously given level, $\bar{G}+G_{-i}$. This possibility differs from what is feasible in standard models of privately provided public goods. Note that the allocation $\left(w_{i} / p, \bar{G}+G_{-i}\right)$ is the point at which the individual has positive direct provision but makes a net contribution of zero. This can be interpreted as the point where, for example, enough carbon offsets are purchased so that the individual's demand for driving (which causes carbon dioxide emissions) is carbon neutral. Although not shown in the figure, other possibilities are for $G_{-i}<0$ and for the feasible set to include levels of $G$ that are below $\bar{G}$. It is assumed, however, that $\bar{G}-\beta \sum_{i=1}^{n} w_{i} \geq 0$. This is sufficient to guarantee that $\bar{G}+G_{-i}-\beta w_{i} \geq 0$ for all $i$, which eliminates the complication of having to contend with negative quantities of the public good.

The unique solution to (2) can be written as

$$
G=\max \left\{f_{i}\left(p, m_{i}\right), \bar{G}+G_{-i}-\beta w_{i}\right\},
$$


where $f_{i}\left(p, m_{i}\right)$ is the demand function for $G$ ignoring the inequality constraint in (2), and $m_{i}=w_{i}+\bar{G}+G_{-i}$ is full income. Subtracting $\bar{G}+G_{-i}$ from both sides, each individual's optimal net contribution, or best response function, is

$$
g_{i}=\max \left\{f_{i}(p, m)-\bar{G}-G_{-i},-\beta w_{i}\right\} .
$$

The final element of the setup is the standard normality assumption in models of privately provided public goods. The assumption simply requires that, for all individuals, both the private and public goods are normal with respect to full income. In particular, the assumption requires that $0<\partial f_{i} / \partial m_{i} \leq \mu<1$ for all $i$ and some constant $\mu .^{7}$

A Nash equilibrium in the model is a set of strategies $\left\{x_{i}^{*}, y_{i}^{*}\right\}_{i=1}^{n}$ that satisfies (1) for all $i$. Using the identity that $g_{i}^{*}=y_{i}^{*}-\beta x_{i}^{*}$ and the budget constraint, it is straightforward to show that this definition is equivalent to a set of net contributions $\left\{g_{i}^{*}\right\}_{i=1}^{n}$ that satisfies $G^{*}=\bar{G}+\sum_{i=1}^{n} g_{i}^{*}$. The latter definition is used to prove the following proposition. ${ }^{8}$

Proposition 1 There exists a unique Nash equilibrium.

The equilibrium level of the public good $G^{*}$ can be either greater than or less than the initial, ambient level $\bar{G}$. Either case is possible because nothing prevents the sum of net contributions $\sum_{i=1}^{n} g_{i}^{*}$ from being positive or negative.

It is worth emphasizing that the Nash equilibrium will not, in general, be Pareto efficient. As in the standard model of a privately provided public good, efficient provision is defined by the Samuelson condition, which requires that the sum of individual marginal rates of substitution equals the marginal rate of transformation (i.e., the price ratio in this case). In contrast, the Nash equilibrium, which is consistent with the solution to (1) for all individuals, is based on individual comparisons between their own marginal rate of substitution and the price ratio; they are equal for those making direct donations, while other individuals are at a corner solution. In this regard, the only difference in the model presented here is that the implicit price ratio is a function of the technology parameter $\beta$.

\section{Direct Provision and Net Contributions}

We can solve for each individual's equilibrium level of direct provision and net contribution. In doing so, it is useful to parameterize heterogenous preferences

\footnotetext{
${ }^{7}$ The parameter $\mu$ is part of the normality assumption to place an upper bound on the equilibrium level of the public good as $n$ grows to infinity. In all of the analysis that follows, only the results in section 6 rely on this part of the assumption.

${ }^{8}$ All proofs, with the exception of that for proposition 2 , which is immediate from the text, are included in the Appendix.
} 


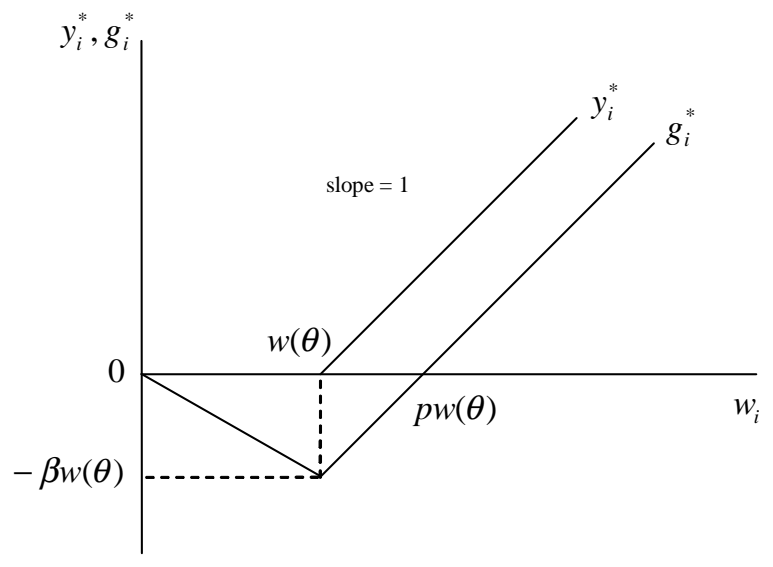

Figure 2: Individuals' direct provision and net contribution

with a variable $\theta$ that indicates an individual's type. Thus, utility functions are written as $U_{i}=U\left(x_{i}, G ; \theta\right)$, and without loss of generality, $\theta$ can be defined such that greater values imply greater demand for $G$.

It must hold that $G^{*}=f\left(p, w_{i}+\bar{G}+G_{-i}^{*} ; \theta\right)$ for all individuals at an interior solution. Taking the inverse and adding $g_{i}^{*}$ to both sides, we can solve for $g_{i}^{*}=$ $w_{i}-p w(\theta)$, where $w(\theta)=\left(f^{-1}\left(G^{*} ; \theta, p\right)-G^{*}\right) / p$. To solve for $y_{i}^{*}$ for these same individuals, use the identity $y_{i}^{*}=g_{i}^{*}+\beta x_{i}^{*}$ and substitute in $g_{i}^{*}$ and the budget constraint to find that $y_{i}^{*}=w_{i}-w(\theta)$. Combining these two results, along with the possibility for corner solutions, proves the next proposition.

Proposition 2 In equilibrium, each individual i's direct provision and net contribution will be

$$
y_{i}^{*}= \begin{cases}w_{i}-w(\theta) & \text { if } w_{i}>w(\theta) \\ 0 & \text { if } w_{i} \leq w(\theta)\end{cases}
$$

and

$$
g_{i}^{*}= \begin{cases}w_{i}-p w(\theta) & \text { if } w_{i}>w(\theta) \\ -\beta w_{i} & \text { if } w_{i} \leq w(\theta)\end{cases}
$$

where $w(\theta)$ is a critical level of wealth that is decreasing in type $\theta$.

Figure 2 illustrates proposition 2 for a given type $\theta$. Note that the expression for direct provision mirrors that for the standard model of private provision of a public good (Andreoni 1988). If wealth is below the critical level, there is no direct provision, but if wealth is above the critical level, direct provision equals the difference $w_{i}-w(\theta)$. Net contributions can be negative but are constrained such that $g_{i}^{*} \geq-\beta w_{i}$. We can see that $g_{i}^{*} \geq 0$ if and only if $w_{i} \geq p w(\theta)$, 
Table 1: Equilibria with different $\beta$ and distributions of $\alpha_{i}$

\begin{tabular}{|c|c|c|c|c|c|}
\hline & (1) & $(2)$ & (3) & (4) & $(5)$ \\
\hline & $\alpha_{i}=.5$ & $\alpha_{i} \in(.4, .6)$ & $\alpha_{i} \in(.3, .7)$ & $\alpha_{i} \in(.2, .8)$ & $\alpha_{i} \in(.1, .9)$ \\
\hline \multicolumn{6}{|l|}{$\beta=0$} \\
\hline$G^{*}$ & 99.5 & 136.1 & 191.3 & 288.9 & 429.6 \\
\hline mean $g_{i}^{*}$ & .50 & .68 & .96 & 1.44 & 2.15 \\
\hline mean $y_{i}^{*}$ & .50 & .68 & .96 & 1.44 & 2.15 \\
\hline \# donors & 200 & 20 & 17 & 20 & 19 \\
\hline \multicolumn{6}{|l|}{$\beta=1$} \\
\hline$G^{*}$ & 99.5 & 98.1 & 89.0 & 81.4 & 77.6 \\
\hline mean $g_{i}^{*}$ & .50 & .49 & .45 & .41 & .39 \\
\hline mean $y_{i}^{*}$ & 50.25 & 50.25 & 50.22 & 50.20 & 50.19 \\
\hline \# donors & 200 & 200 & 199 & 173 & 151 \\
\hline \multicolumn{6}{|l|}{$\beta=2$} \\
\hline$G^{*}$ & 99.5 & 98.1 & 89.0 & 76.29 & 61.0 \\
\hline mean $g_{i}^{*}$ & .50 & .49 & .44 & .38 & .31 \\
\hline mean $y_{i}^{*}$ & 66.83 & 66.83 & 66.81 & 66.79 & 66.77 \\
\hline \# donors & 200 & 200 & 200 & 200 & 183 \\
\hline
\end{tabular}

which is the critical level of wealth at which direct provision exactly offsets the negative affect on $G$ that comes from the individual's consumption of $x_{i}$ (e.g., the point where an individual is exactly carbon neutral). At lower levels of wealth, individual's have a negative affect on $G$. At sufficiently low levels of wealth, $g_{i}^{*}$ is decreasing with slope $-\beta$; whereas, at intermediate levels of wealth, $g_{i}^{*}$ is still negative but increasing with slope one, as direct provision begins to be offsetting.

To see the effect of heterogenous preferences, consider two different types $\theta_{h}>\theta_{l}$ with the same level of wealth. The respective critical levels of wealth will be $w\left(\theta_{h}\right)<w\left(\theta_{l}\right) .{ }^{9}$ Thus, individuals with a greater $\theta$ will begin direct provision at a lower level of wealth. At sufficiently high levels of wealth, both $y_{h}^{*}>y_{l}^{*}$ and $g_{h}^{*}>g_{l}^{*}$; but at sufficiently low levels of wealth, neither type will engage in direct provision, and they will have the same negative effect on the public good.

In order to further examine the effects of heterogeneity on the Nash equilibrium, it is useful to consider an example. Assume there are $n=200$ individuals, each with an endowment $w=100$ and Cobb-Douglas preferences of the form $U_{i}=x_{i}^{\alpha_{i}} G^{1-\alpha_{i}}$. Table 1 characterizes the Nash equilibria under different assumptions about $\beta$ and the distribution of $\alpha_{i}$. In all cases, $\bar{G}=0$ and the outcomes are reported for $G^{*}$, mean $g_{i}^{*}$, mean $y_{i}^{*}$, and the number of individuals making a

\footnotetext{
${ }^{9}$ The inequality follows by construction. The variable $\theta$ is defined such that $f\left(p, m ; \theta_{h}\right)>$ $f\left(p, m ; \theta_{l}\right)$. Thus, $f^{-1}\left(G^{*} ; \theta_{h}, p\right)<f^{-1}\left(G^{*} ; \theta_{l}, p\right)$, and this implies that $w\left(\theta_{h}\right)<w\left(\theta_{l}\right)$.
} 
direct donation. Column (1) refers to the case in which $\alpha_{i}=.5$ for all $i$, while the other columns refer to cases in which $\alpha_{i}$ is randomly generated from a uniform distribution within the indicated interval. The first scenario, with $\beta=0$, is consistent with the standard model of a privately provided public good. It is clear in this case that greater heterogeneity increases provision of the public good while decreasing the number of donors. Note that the same pattern does not hold in the cases where $\beta>0 .{ }^{10}$ With greater heterogeneity, $G^{*}$ changes relatively little, and the number of donors decreases far less quickly. This is due to the fact that consumption of $x_{i}$ is no longer independent of $G$, so the effect of having more individuals with a stronger preference for the public good is attenuated by also having more individuals with a weaker preference. As some individuals are more likely to favor private consumption, they also diminish the level of the public good.

\section{Comparative Statics}

This section considers comparative static properties of the Nash equilibrium. The analysis focuses on the ways in which changes in the exogenous variables affect the equilibrium level of the public good $G^{*}$. Because none of the results depends on the parameterization of heterogenous preferences, notation for $\theta$ is dropped and preference heterogeneity is captured once again with subscripts $i$.

Let us begin with a change in $\beta$, that is, a change in the technology of the private good as it relates to environmental quality. Referring back to Figure 1, we can see that changes in $\beta$ shift the budget frontier like a change in the price of the private good, as $p=1+\beta$. It follows that, for interior solutions, an individual's demand for $G$ satisfies $\partial f_{i} / \partial \beta=\partial f_{i} / \partial p$, and the sign of the effect will be $<(>)$ zero if $G$ is a gross complement (substitute) for $x_{i}$. For corner solutions, however, there is only one possibility, as demand for the public good is $G=\bar{G}+G_{-i}-\beta w_{i}$, which is decreasing in $\beta$. Combining these results, it follows that an individual's demand for $G$ is decreasing in $\beta$ if either $G$ is a gross complement for $x_{i}$ or the individual is at a corner solution (i.e., $y_{i}=0$ ). In contrast, an individual's demand for $G$ is increasing in $\beta$ if $G$ is a gross substitute for $x_{i}$ and the individual is at an interior solution (i.e., $y_{i}>0$ ). The next proposition shows how these comparative statics for an individual's demand translate into equilibrium results. ${ }^{11}$

Proposition 3 For all $\beta \geq 0$, a change in the technology will affect the equilibrium level of the public good $G^{*}$ such that (a) $d G^{*} / d \beta<0$ if either $G$ is a gross

\footnotetext{
${ }^{10}$ The level of provision $G^{*}$ is the same for all cases in column (1) because demand for $G$ does not depend on $\beta$ (i.e., the price of $x_{i}$ since $p=1+\beta$ ) with Cobb-Douglas preferences.

${ }^{11}$ Although the setup of the models is different, the mechanism underlying these results is closely related to that in Cornes and Sandler (1989) and Kotchen (2006).
} 


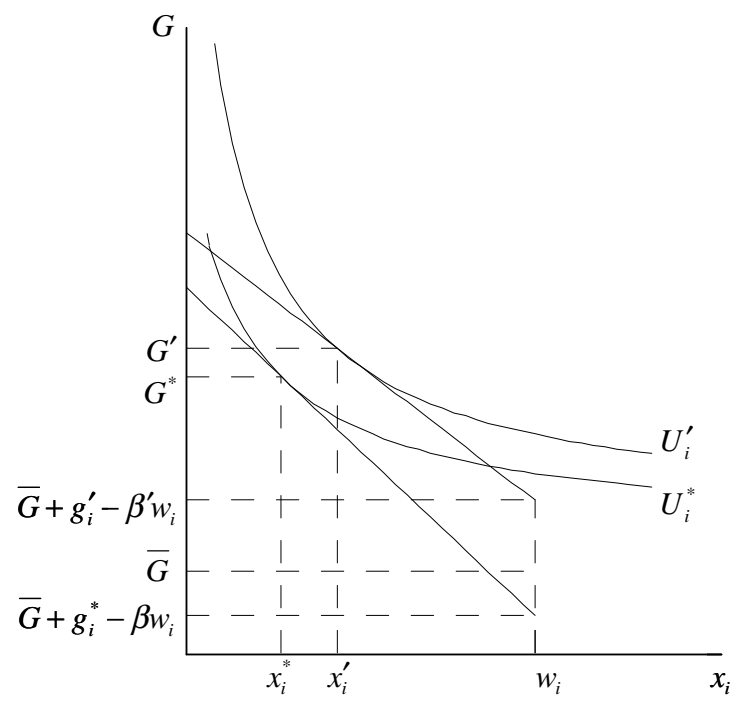

Figure 3: Two identical individuals with $U_{i}=\left(x_{i}^{-.9}+G^{-.9}\right)^{-1 / .9}, w_{i}=50$,

$$
\bar{G}=10, \beta=.35 \text { and } \beta^{\prime}=.1 \text {. }
$$

complement for $x_{i}$ for all $i$ or $y_{i}^{*}=0$ for all $i$; or (b) $d G^{*} / d \beta>0$ if $G$ is a gross substitute for $x_{i}$ and $y_{i}^{*}>0$ for all $i$.

Consider a case in which there is a decrease in $\beta$, that is, the private good becomes more environmentally friendly. One result is that private consumption (weakly) increases because it is now relatively less costly. But the effect on demand for environmental quality will depend on whether $G$ is a gross complement or substitute for $x_{i}$. In the former case, environmental quality will unambiguously increase, but in the later case, environmental quality can decrease. Figures 3 and 4 show examples of each, respectively. In both cases, the equilibrium level of the public good changes from $G^{*}$ to $G^{\prime}$.

It is worth emphasizing that the possibility for a decrease in $G$ is rather counterintuitive: a change in the technology that makes the private good more "green" has a detrimental effect on environmental quality. The reason is that in addition to stimulating demand for private consumption, it also decreases demand for the public good if $G$ is a gross substitute for $x_{i}$. Hence the decrease in direct donations can outweigh the environmental benefits of the change in technology. As the proposition suggests, however, beneficial effects on the environment are more likely if environmental quality is a gross complement for private consumption and if the economy has a larger proportion of individuals that do not engage in direct provision. 


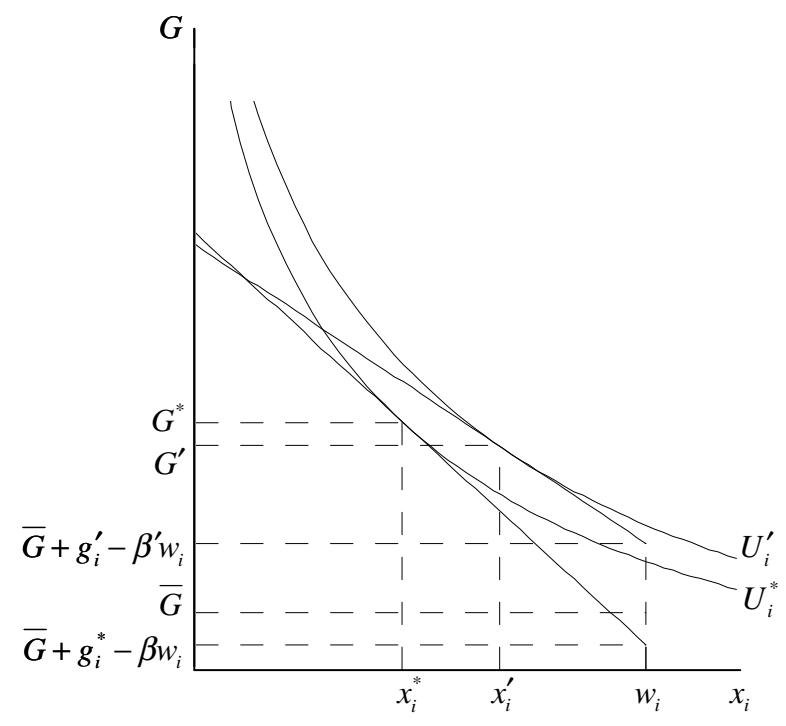

Figure 4: Two identical individuals with $U_{i}=\left(x_{i}^{4}+G^{\cdot 4}\right)^{1 / .4}, w_{i}=50, \bar{G}=10$,

$$
\beta=.45 \text { and } \beta^{\prime}=.05 \text {. }
$$

Another way of interpreting this same result is that an increase in $\beta$, which implies a less green technology, can increase environmental quality. Figure 4 demonstrates this possibility with the change (in reverse) from $G^{\prime}$ to $G^{*}$. The intuition is that private consumption becomes more costly, and because $G$ is a gross substitute for $x_{i}$, demand for the public good increases.

Now consider a change in $\bar{G}$, the ambient level of the public good. A change in $\bar{G}$ will affect each individual's full income, and because $G$ is normal, it is intuitive that a change in $\bar{G}$ will affect demand for $G$ in the same direction. This reasoning underlies the next equilibrium result.

Proposition $4 A$ change in $\bar{G}$ will affect the level of the public good $G^{*}$ such that $0<d G^{*} / d \bar{G} \leq 1$.

The fact that $d G^{*} / d \bar{G} \leq 1$ reflects the further assumption that $x_{i}$ is normal for all $i$. An increase in $\bar{G}$, for example, must stimulate demand for $x_{i}$ as well. The weak inequality accounts for the possibility that all individuals are at a corner solution, in which case $d G^{*}=d \bar{G}$. A general interpretation of proposition 4 is that, given changes in the ambient level of the public good, there will be (weak) crowding-out of private provision, but the crowding-out will be less than one-for-one.

A change in $w_{i}$ for some $i$ has the initial effect of changing the individual's full income, but the effect on the level of the public good will depend on whether the individual engages in direct provision. 
Proposition 5 A change in $w_{i}$ for some $i$ will affect the equilibrium level of the public good $G^{*}$ such that (a) $0<d G^{*} / d w_{i}<1$ if the inequality constraint in (2) is not binding for individual $i$; or (b) $-\beta \leq d G^{*} / d w_{i}<0$ if the constraint is binding.

The intuition underlying proposition 5 is easy to see in the special case where $n=1$. If the individual starts at an interior solution, the normality assumption implies that an increase in $w_{i}$ will increase demand for $G$. In contrast, if the individual starts at a corner solution, an increase in $w_{i}$ will decrease demand for $G$. The other bounds on both results are due to the fact that with $n \geq 2$, there is crowding out in response to individual $i$, but it will be incomplete. Note that the general result here differs from the standard model of private provision of a public good, in which case the normality assumption implies that the equilibrium level of the public good must be nondecreasing in any individual's endowment.

One respect in which the extension considered here does not differ from the standard model is the neutrality result. ${ }^{12}$ This result focuses on redistributions of income and states that total provision of the public good is invariant to any income redistribution that leaves the set of donors unchanged. To see how the result continues to hold here, let $D$ denote the set donors and use proposition 2 to write

$$
G^{*}=\bar{G}+\sum_{i=1}^{n} g_{i}^{*}=\bar{G}+\sum_{i \in D}\left[w_{i}-p w(\theta)\right]-\sum_{i \notin D} \beta w_{i} .
$$

The neutrality result then follows immediately upon recognizing that $w(\theta)$ is independent of the income distribution as long as the set of donors does not change. A further and well-known implication of this same result relates to crowding-out. Specifically, government provision, which is financed through taxes on donors that do not exceed any individual's donation, will crowd-out private provision one-for-one, leaving the level of the public good unchanged.

\section{Social Welfare}

A standard result in the public goods literature is that the private provision equilibrium is not Pareto optimal, and as described previously, this result applies here as well. But how will changes in the exogenous parameters affect social welfare? This section expands on the comparative static analysis to consider its welfare implications.

The effects of a change in $\bar{G}$, which are the most straightforward, are unambiguous: an increase in the ambient level of the public good, for example,

\footnotetext{
${ }^{12}$ See Warr (1983) for the original formulation and Bergstrom, Blume and Varian (1986) for a generalization.
} 
results in a Pareto improvement. We have shown already that the level of the public good increases and that crowding out is less than one-for-one. Together, these results imply that every individual's full income must increase, and this unambiguously makes everyone strictly better off.

Proposition 6 In equilibrium, a change in $\bar{G}$ will affect each individual's utility such that $d U_{i} / d \bar{G}>0$ for all $i$.

The effect of a change in $w_{i}$ for some $i$ depends once again on whether or not individual $i$ engages in direct provision. If, for example, the individual spends some increased endowment on direct provision, then the level of public good increases, and everyone is made better off. But if the increased endowment is spent on private consumption, then the level of the public good decreases, causing the individual to be better off, while everyone else is worse off.

Proposition 7 In equilibrium, if the inequality constraint in (2) is not binding for individual $i$, then $d U_{j} / d w_{i}>0$ for $j=1, \ldots, n$; however, if the inequality constraint is binding for individual $i$, then $d U_{i} / d w_{i}>0$ while $d U_{j} / d w_{i}<0$ for all $j \neq i$.

Referring back to the examples in figures 3 and 4, we can see how a decrease in $\beta$ can result in a Pareto improvement, regardless of whether $G^{*}$ increases or decreases. What is perhaps a more counterintuitive result is that a decrease in $\beta$ can actually make all individuals worse off. That is, making a private good more environmentally friendly can actually decrease social welfare. Figure 5 shows an example in which the level of the public good decreases and causes a negative income effect that outweighs the positive income effect from the reduction in $\beta$ (i.e., in the price of $x_{i}$ ). The result is that utility decreases for both individuals.

In general, heterogeneity of preferences and endowments means that a change in $\beta$ can make some individuals better off and others worse off, regardless of whether $G$ is a gross complement or substitute for $x_{i}$. But if we consider the special case in which all individuals have identical preferences and endowments, we can get traction on the features that are more likely to drive the results one way or the other. The next proposition implies that, for example, making the private good more environmentally friendly is more likely to be welfare improving if $G$ is a gross complement for $x_{i}$ and/or if more individuals do not make a direct donation. ${ }^{13}$

\footnotetext{
${ }^{13}$ The approach taken to prove this result follows that in Cornes and Sandler (1989), and the fundamental insight is closely related.
} 


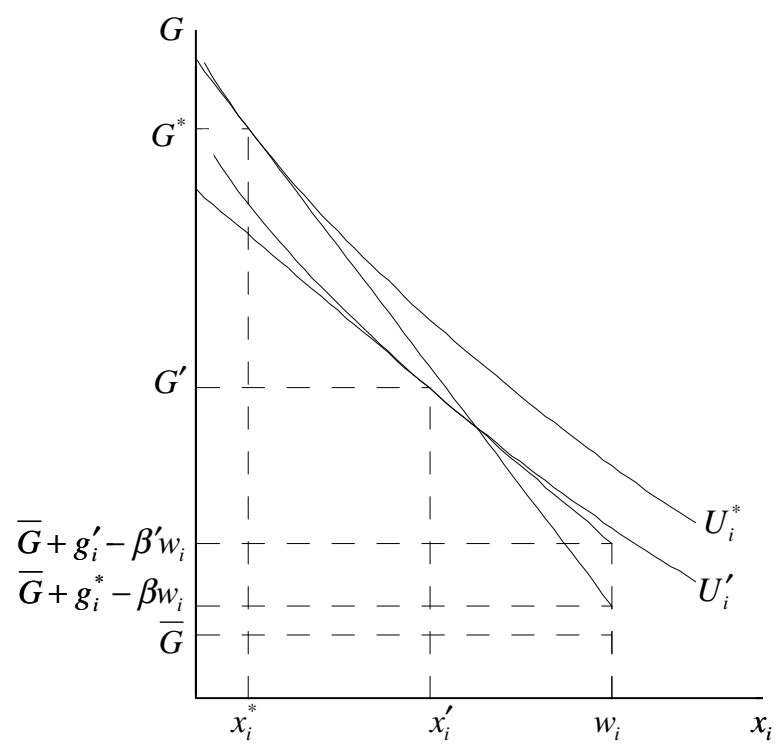

Figure 5: Two identical individuals with $U_{i}=\left(x_{i}^{.8}+G^{.8}\right)^{1 / .8}, w_{i}=50, \bar{G}=10$, $\beta=.7$ and $\beta^{\prime}=.1$.

Proposition 8 Given an economy of $n \geq 2$ identical individuals with endowments $w$, a change in $\beta$ will affect each individual's level of utility such that

$$
\frac{d U}{d \beta}=\left\{\begin{array}{ll}
\left(\frac{\partial f / \partial p}{n /(n-1)-\partial f / \partial m}-x_{i}^{*}\right) / \frac{\partial U}{\partial G} & \text { if } w>w^{*} \\
-n w \frac{\partial U}{\partial G} & \text { if } w \leq w^{*}
\end{array},\right.
$$

where $w^{*}$ is the critical level of wealth defined in proposition 2.

If individuals are at a corner solution, a decrease in $\beta$ reduces each individual's negative externality, and the result is a Pareto improvement. ${ }^{14}$ If, however, individuals are at an interior solution, the welfare effects depend on the sign of the first expression in (4). The fact that it is negative only if $\partial f / \partial p<0$ implies that $G$ being a gross substitute for $x_{i}$ is necessary but not sufficient for $d U / d \beta>0$. Equation (4) also implies that welfare immiserization is more likely with lower initial consumption of $x_{i}$, greater $n$, a larger "spillin effect" captured by $\partial f / \partial m$, and a larger "price effect" captured by $\partial f / \partial p$. Note that a larger price effect is consistent with a greater elasticity of substitution, as the contrast between the curvature of the indifference curves in figures 4 and 5 suggests.

\footnotetext{
${ }^{14}$ It is straightforward to show that this result holds even with heterogeneous preferences and endowments.
} 
Although we know that the equilibrium quantity of the public good will not, in general, be Pareto efficient, it is worth considering how changes in $\beta$ may affect the extent of underprovision. Cornes and Sandler's (1996) index of "easy riding" provides a useful way to quantify underprovision of a public good. The index is simply the ratio of equilibrium provision over efficient provision, where the latter is based on the optimum that is consistent with the shares of individual contributions implied by the equilibrium. Continuing to assume $n$ identical individuals with the same endowments, let us consider CES preferences of the form $U_{i}=\left(x_{i}^{\rho}+G_{i}^{\rho}\right)^{\frac{1}{\rho}}$. In this case, it is straightforward to solve for the index of easy riding

$$
\lambda=\frac{1+(n p)^{r}}{1+n p^{r}},
$$

where $r=\rho /(\rho-1)$ and it still holds that $p=1+\beta$. Because $r<1$ for all permissible values of $\rho<1$, it follows that $\lambda<1$ for all $n \geq 2$. Now, differentiating (5) with respect to $\beta$ and rearranging, we find that $\partial \lambda / \partial \beta=\partial \lambda / \partial p$ will have the opposite sign of $r .{ }^{15}$ Hence $\partial \lambda / \partial \beta>(<) 0$ if $G$ and $x_{i}$ are gross substitutes (complements). In this case, therefore, changes in $\beta$ that increase (decrease) voluntary provision also decrease (increase) the gap between equilibrium and efficient provision. While this result need not apply more generally, the preceding analysis demonstrates how, given interior solutions, understanding the effects of changes in $\beta$ on social welfare are equivalent to understanding the effects of changes in the price of private consumption in the standard public good model.

\section{Offsets in a Large Economy}

In general, the number of individuals in the economy will affect the equilibrium level of a privately provided public good. Two fundamental results for the pure public good model are that, as $n$ increases to infinity, the aggregate level of the public good increases to a finite level and the average level of individual provision decreases to zero (Andreoni 1988). These results do not apply, however, if direct provision is used to compensate for provision of a public bad that comes from consumption of another good. In such a model, Vicary (2000) shows that the mean level of direct provision converges to a positive value, while mean net contributions converge to zero. While the model developed here produces a result similar to that in Vicary (2000), other results are new and directly comparable to those in Andreoni (1988).

\footnotetext{
${ }^{15}$ The partial derivative simplifies to $\frac{\partial \lambda}{\partial \beta}=\frac{r n p^{r-1}}{1+n p^{r}}\left(n^{r-1}-1\right)$. The term in parentheses is always nonpositive because $r<1$; therefore, the overall expression will be positive (negative) if $r$ is negative (positive). A standard result for the CES utility function, moreover, is that the two goods are gross substitutes (complements) if $r$ less than (greater than) zero.
} 
Consider again the special case in which all individuals have identical preferences and endowments. The next proposition summarizes the important properties of the Nash equilibrium as the number of individuals in the economy grows large.

Proposition 9 Given an economy of $n$ identical individuals with endowments $w$, as $n \rightarrow \infty, G^{*}$ converges to a strictly positive finite level, each individual's net contribution $g^{*} \rightarrow 0$, and each individuals direct provision $y^{*} \rightarrow w \beta / p$.

This special case is sufficient to show the key result: direct provision remains positive while net contributions converge to zero. To build intuition, consider what would happen if, to the contrary, direct provision were zero. The level of the public good would continue to decrease as individuals were added; however, this could not continue indefinitely because the public good is normal. Hence there exists a sufficiently large $n$ such that direct provision must occur. It follows that, because the public good is nonrival and nonexcludable, $G$ converges to a finite positive level, which implies that direct provision must converge to a point where it exactly offsets the negative effect that occurs via consumption of the private good. Again, this is the point at which, for example, an individual concerned with climate change would purchase enough offsets to be carbon neutral. The result is reflected by the way that $y^{*} \rightarrow w \beta / p$, which is the negative effect that each individual imposes on the public good when the the full cost of $x_{i}$ (in terms of $G$ ) is taken into account. ${ }^{16}$

A final observation pertains to the trajectory of $G^{*}$ as $n \rightarrow \infty$. In the standard pure public good model, the level of the public good increases monotonically as the economy grows large. But this result need not apply here. It was noted in section 2 that, for any $n$, the level of $G^{*}$ can be greater than or less than the initial level $\bar{G}$. It follows that, as $n$ increases, the equilibrium level of the public good can be either increasing or decreasing, depending on how demand for $G$ compares to $\bar{G}$. While it can be shown that the trajectory will be monotonic with identical individuals, even this need not hold in an economy with heterogenous preferences or endowments.

\section{Conclusion}

This paper examines an additional motivation for why individuals voluntarily provide public goods: to offset other activities that have a negative effect on the level of the public good. An important result, related to one shown previously,

\footnotetext{
${ }^{16}$ Vicary's (2000) main result is that direct provision converges to a positive value, but he does not solve for the value to which it converges.
} 
is that direct provision of the public good will persist even in large economies, as the incentive for offsets grows even stronger. Other results are new and comparable to those from the standard model of private provision of a pure public good. The Nash equilibrium is solved explicitly to show how individual direct donations and net contributions depend on wealth and heterogenous preferences. The comparative static analysis demonstrates how the equilibrium level of the public good and social welfare depend on the technology, individual wealth, and the initial level of the public good. The possible effects of changes in technology are rather counterintuitive, as a technological improvement can either increase or decrease provision of the public good and social welfare.

Application of the model in an environmental context establishes a starting point for understanding and making predictions about markets such as those for carbon offsets. The model shows how some individuals will not purchase offsets; and while other individuals will, they can still have either a positive or negative effect on emissions. The special case of carbon neutrality is highlighted in the context of more general possibilities. Implications of the comparative static analysis relate to the possible effects of technological innovations such as more fuel efficient vehicles. The overall effect that such innovations will have on emissions will depend on how they change the incentives for offsets. Important factors to consider are the proportion of individuals that purchase offsets and whether demand for the private good is a complement or substitute for concern about climate change. Finally, the model provides a theoretical explanation for why we see the emergence of markets for individual purchases of carbon offsets despite the fact that climate change is such a large and growing problem.

A possible direction for future research is to consider the consequences of combining the incentive for offsets with other motives for voluntary provision of a public good. With environmental offsets, for example, it is easy to see how motives associated with social approval or signalling could be important. This would explain why those selling carbon offsets for vehicles typically provide window decals and bumper stickers that enable customers to advertise the fact that they purchased an offset. At the same time, people might obtain a feeling of warm-glow satisfaction from the act of purchasing an offset, but this raises the further question of whether they also feel guilty about being responsible for pollution. Future research that systematically addresses these topics would further our understanding of voluntary provision of public goods in general and environmental offsets in particular. 


\section{Appendix}

\section{Proof of Proposition 1}

Define the function $h_{i}\left(w_{i}, \beta, \bar{G}+G_{-i}\right)=\max \left\{f_{i}\left(p, m_{i}\right), \bar{G}+G_{-i}-\beta w_{i}\right\}$. Let $a_{i}=h_{i}\left(w_{i}, \beta, \bar{G}-\beta \sum_{j \neq i} w_{j}\right)$, which is individual $i$ 's demand for the public good if all other individuals spend their wealth on the private good only. For $G \geq a_{i}$, define $r_{i}$ implicitly with $G=h_{i}\left(w_{i}, \beta, G-r_{i}\right)$. Thus, $G-r_{i}=\bar{G}+G_{-i}$ is the exogenously given level of the public good that causes individual $i$ to demand $G$ and make net contribution $g_{i}=r_{i}$. By the implicit function theorem, $r_{i}=r_{i}\left(w_{i}, \beta, G\right)$ is a well-defined and continuous function. By definition $r_{i}\left(w_{i}, \beta, a_{i}\right)=a_{i}-\bar{G}+\beta \sum_{j \neq i} w_{j}$, and $\partial r_{i} / \partial G=1-1 /\left(\partial h_{i} / \partial G_{-i}\right) \leq 0$. Now define $a=\max \left\{a_{i}\right\}_{i=1}^{n}$. For $G \geq a$, let $R(\mathbf{w}, \beta, G)=\sum_{i=1}^{n} r_{i}$. Clearly, $R$ is continuous and $\partial R / \partial G \leq 0$. It is also true that $R(\mathbf{w}, \beta, a)+\bar{G} \geq a$. This follows because the left hand side can be rewritten as $a+\sum_{j \neq i}\left[\beta w_{j}+r_{j}\left(w_{j}, \beta, a\right)\right]$, where individual $i$ is the one for whom $a_{i}=a$, and it must hold that $r_{j} \geq-\beta w_{j}$ for all $j$. Hence, by the intermediate value theorem and monotonicity of $R$, there exists a unique $G^{*}$ that satisfies $R\left(\mathbf{w}, \beta, G^{*}\right)+\bar{G}=G^{*}$, and $g_{i}^{*}=r_{i}\left(w_{i}, \beta, G^{*}\right)$ specifies a unique equilibrium strategy for all $i$. $Q E D$

\section{Proof of Propositions 3, 4, and 5}

The general approach is to begin with the equilibrium condition that is established in the proof of proposition 1: $R\left(\mathbf{w}, \beta, G^{*}\right)+\bar{G}=G^{*}$. Differentiating with respect to a change in any one of the exogenous variables, we can solve for

$$
\frac{d G^{*}}{d k}=\left(\frac{\partial R}{\partial k}+\frac{d \bar{G}}{d k}\right) /\left(1-\frac{\partial R}{\partial G^{*}}\right) .
$$

The task, then, is to determine the sign of this expression for $k=\beta, \bar{G}$, or $w_{i}$ for some $i$. We have already shown that $\partial R / \partial G^{*} \leq 0$, so the denominator is greater than or equal to one in all cases. It is clear that if $k=\bar{G}$, then $d \bar{G} / d k=1$, otherwise $d \bar{G} / d k=0$. Thus, the sign of (6) for each $k$ will depend on $\partial R / \partial k$.

Prop. 3-Solving for $\partial r_{i} / \partial \beta$ and summing implies that

$$
\partial R / \partial \beta=\sum_{i=1}^{n}\left[\left(\partial h_{i} / \partial \beta\right) /\left(\partial h_{i} / \partial \bar{G}_{-i}\right)\right],
$$

where $\bar{G}_{-i}=\bar{G}+G_{-i}$. Using the definition of $h_{i}$ the result follows immediately from equation (6), the normality assumption, and the implications of $G$ being either a gross compliment or substitute for $x_{i}$ for all $i$.

Prop 4.- Holding $G$ constant while changing $\bar{G}$ implies that $\partial r_{i} / \partial \bar{G}=0$, which implies that $\partial R / \partial \bar{G}=0$. This result combined with equation (6) proves proposition 4. 
Prop. 5-If the inequality constraint in (2) is not binding for individual $i$, then $\partial r_{i} / \partial w_{i}=\partial R / \partial w_{i}=1$ and $\partial R / \partial G^{*}<0$. These facts combined with $(6)$ prove the first part of proposition 5 . If the inequality constraint is binding for individual $i$, then $\partial r_{i} / \partial w_{i}=\partial R / \partial w_{i}=-\beta$, and this combined with (6) proves the second part of proposition 5. QED

\section{Proof of Proposition 6}

Because utility is strictly increasing in $m_{i}$, it is sufficient to show that $d m_{i}^{*} / d \bar{G}>0$ for all $i$. By definition $d m_{i}^{*} / d \bar{G}=d G_{-i}^{*} / d \bar{G}+1$. Taking the differential of equation (3), solving for $d G_{-i}^{*} / d \bar{G}$, and substituting it in implies that $d m_{i}^{*} / d \bar{G}=$ $\left(d G^{*} / d \bar{G}\right) /\left(\partial f_{i} / \partial m_{i}\right)$ or $d m_{i}^{*} / d \bar{G}=d G^{*} / d \bar{G}+1$ for an interior or corner solution, respectively. The normality assumption and proposition 4 imply that both expressions are strictly greater then zero. $Q E D$

\section{Proof of Proposition 7}

We can prove the results in terms of changes in $d m_{j}^{*} / d w_{i}$ for $j=1, \ldots, n$. By definition $d m_{j}^{*} / d w_{i}=d G_{-j}^{*} / d w_{i}+d w_{j} / d w_{i}$. Taking the differential of equation (3), solving for $d G_{-j}^{*} / d w_{i}$, and substituting it in implies that $d m_{j}^{*} / d w_{i}=$ $\left(d G^{*} / d w_{i}\right) /\left(\partial f_{j} / \partial G_{-j}\right)$ if the inequality constraint is not binding; otherwise $d m_{j}^{*} / d w_{i}=\left(d G^{*} / d w_{i}\right)+\left(d w_{j} / d w_{i}\right)(1+\beta)$. The proposition follows immediately from these expressions combined with the normality assumption and proposition 4. $Q E D$

\section{Proof of Proposition 8}

If $w<w^{*}$, each individual's utility is $U_{i}=U(w, \bar{G}-n \beta w)$. Take the differential and solve for $d U / d \beta$ to get the first expression in (4). If $w \geq w^{*}$, then $G^{*}=f\left(1+\beta, w+\bar{G}+(n-1) G^{*} / n\right)$. Take the differential and solve for $d G^{*} / d \beta=(\partial f / \partial p) /[1-(\partial f / \partial m) n /(n-1)]$. Next, take the differential of the budget constraint and solve for $d x_{i}^{*}=-\left(x_{i}^{*} d \beta+d g_{i}^{*}\right) / p$. Also, take the differential of $U_{i}=U\left(x_{i}^{*}, G^{*}\right)$ and use the fact that $U_{x_{i}} / U_{G}=p$ to solve for $d U_{i}=(\partial U / \partial G)\left(p d x_{i}^{*}+d G^{*}\right)$. Finally, substitute $d x_{i}^{*}$ and $d G^{*} / d \beta$ into $d U_{i}$ and solve for $d U / d \beta$ to get the second expression in (4). $Q E D$

\section{Proof of Proposition 9}

Let $G_{n}^{*}$ denote the equilibrium level of the public good, and let $w_{n}^{*}$ denote the critical level of wealth defined in proposition 2. If $w \leq w_{n}^{*}$, then $G_{n}^{*}=\bar{G}-n \beta w \geq$ $f(p, w+\bar{G}-(n-1) \beta w)$. As $n$ increases, the left hand side of the inequality decreases at rate $-\beta w$ and the right hand side at rate $-(\partial f / \partial m) \beta w$. Thus, by 
the normality assumption, there exists a sufficiently large $n$ such that $w \leq w_{n}^{*}$ can no longer hold, and it must be true that $w>w_{n}^{*}$. In this case, $w_{n}^{*}=$ $\left(f^{-1}\left(G_{n}^{*}\right)-G_{n}^{*}\right) / p$, which can be written more compactly as $w_{n}^{*}=\phi\left(G_{n}^{*}\right)$. Taking the inverse, we have $G_{n}^{*}=\phi^{-1}\left(w_{n}^{*}\right)$, which is finite because $w>w_{n}^{*}$ and the normality assumption implies that $0<d \phi^{-1} / d w \leq \mu p /(1-\mu)$. By proposition $2, G_{n}^{*}=\bar{G}+n\left(w-p w_{n}^{*}\right)$, which can be rewritten as $\left(\phi^{-1}\left(w_{n}^{*}\right)-\bar{G}\right) / n=w-p w_{n}^{*}$. It follows that, as $n \rightarrow \infty$, we have $w_{n}^{*} \rightarrow w / p$. Hence $g_{n}^{*} \rightarrow 0$ and $y_{n}^{*} \rightarrow w \beta / p$, and we have $G_{n}^{*} \rightarrow \phi^{-1}(w / p)>0$. QED 


\section{References}

Andreoni, James. (1988) "Privately Provided Public Goods in a Large Economy: The Limits of Altruism." Journal of Public Economics 35, 57-73.

Andreoni, James. (1990) "Impure Altruism and Donations to Public Goods: A Theory of Warm-Glow Giving." Economic Journal 100, 464-77.

Bergstrom, Theodore C., Blume, Lawrence E., and Varian, Hal R. (1986) "On the Private Provision of Public Goods." Journal of Public Economics 29, 2549 .

Cornes, Richard, and Hartley, Roger. (2007) "Aggregative Public Good Games." Journal of Public Economic Theory 9, 201-19.

Cornes, Richard, and Sandler, Todd. (1984) "Easy Riders, Joint Production, and Public Goods." Economic Journal 94, 580-98.

Cornes, Richard, and Sandler, Todd. (1989) "Public Goods, Growth, and Welfare" Social Choice and Welfare 6, 243-51.

Cornes, Richard, and Sandler, Todd. (1994) "The Comparative Static Properties of the Impure Public Good Model." Journal of Public Economics 54, 403-21.

Cornes, Richard, and Sandler, Todd. (1996) The Theory of Externalities, Public Goods and Club Goods, Second Edition. Cambridge, U. K.: Cambridge University Press.

Dominus, Susan. (2005) "The Zero-Emissions S.U.V.," The New York Times Magazine, December 11.

Glazer, Amihai and Konrad, Kai A. (1986) "A Signalling Explanation for Charity," American Economic Review 86, 1019-28.

Harbaugh, William T. (1998) "What Do Donations Buy? A Model of Philanthropy Based on Prestige and Warm Glow," Journal of Public Economics 67, 269-84.

Hollander, Heinz. (1990) "A Social Exchange Approach to Voluntary Cooperation," American Economic Review 80, 1157-67.

Kotchen, Matthew J. (2005) "Impure Public Goods and the Comparative Statics of Environmentally Friendly Consumption," Journal of Environmental Economics and Management 49, 281-300. 
Kotchen, Matthew J. (2006) "Green Markets and Private Provision of Public Goods," Journal of Political Economy 114, 816-34.

Kotchen, Matthew J. and Moore, Michael R. (in press) "Conservation: From Voluntary Restraint to a Voluntary Price Premium," Environmental and Resource Economics.

Samuelson, Paul A. (1954) "The Pure Theory of Public Expenditure," Review of Economics and Statistics 36, 387-9.

Samuelson, Paul A. (1954) "A Diagramatic Exposition of a Theory of Public Expenditure," Review of Economics and Statistics 37, 350-6.

Vicary, Simon. (2000) "Donations to a Public Good in a Large Economy." European Economic Review 44, 609-18.

Warr, Peter G. (1983) "The Private Provision of a Public Good is Independent of the Distribution of Income," Economics Letters 13, 207-11. 